\title{
External Cavity Tunability of Some NIR Semiconductor Lasers
}

\author{
M. A. Bani ${ }^{1}$, M. Nazeri ${ }^{*}$, H. Abbasi ${ }^{2}$, A. Sajedi ${ }^{1}$ \\ 1. Faculty of Physics, University of Kashan, Kashan, Iran
}

2. Biomedical Laser and Optics Group, Department of Biomedical Engineering, University of Basel, CH-4123 Allschwil, Switzerland

${ }^{(*)}$ E-mail: $\underline{\text { m_nazeri@kashanu.ac.ir }}$

Received: $11 / 07 / 2017 \quad$ Accepted: $11 / 07 / 2018$

DOI: $10.7149 /$ OPA.51.3.50014

\begin{abstract}
:
In this paper, tunability of four near-infrared semiconductor lasers which typically lase at 783nm, 808 $\mathrm{nm}, 892 \mathrm{~nm}$ and $980 \mathrm{~nm}$ is studied. Both Littrow (wavelength dependent beam direction setup) and Littman (wavelength independent beam direction setup) configurations have been used for single mode tuning. In addition, the tunability of $783 \mathrm{~nm}$ laser in dual mode tuning is studied in both Littrow and Litman configurations. The wavelength and the power of the output beam of the laser have been measured. In all four cases, Littrow configuration shows a little better tuning range. In both Littrow and Littman configurations, the wavelengths near the main wavelength of the laser had more power. The highest achieved power of these lasers in the Littrow setup was more than that of the Littman setup. Moreover, the tuning speed and effect of tuning on the bandwidth of the laser were investigated.
\end{abstract}

Key words: External cavity, Littrow configuration, Littman configuration, near IR, tunability.

\section{REFERENCES AND LINKS / REFERENCIAS Y ENLACES}

[1] N. Peyghambarian, S. W. Koch, A. Mysyrowicz, Introduction to semiconductor optics. Prentice-Hall, Inc (1994).

[2] F. J. Duarte, Tunable lasers handbook. Academic Press (1996).

[3] F. J. Duarte, Tunable laser optics. $2^{\text {nd }}$ Ed. CRC Press (2015).

[4] F. J. Duarte, Tunable laser applications. $2^{\text {nd }}$ Ed. CRC press (2009).

[5] C. Ye, T. K. Wei, Tunable external cavity diode lasers, World Scientific (2004).

[6] M. Tani, O. Morikawa, et al. "Generation of terahertz radiation by photomixing with dual- and multiplemode lasers, Semicond," Sci. Tech. 20, 151-163 (2005).

[7] S. Bennetts, G. D. McDonald, K. S. et al. "External cavity diode lasers with $5 \mathrm{kHz}$ linewidth and $200 \mathrm{~nm}$ tuning range at $1.55 \mu \mathrm{m}$ and methods for linewidth measurement," Opt. Exp. 22, 10642-10654, (2014).

[8] K. S. Hardman, S. Bennetts, et al. "Construction and Characterization of External Cavity Diode Lasers for Atomic Physics," J. Vis. Exp. 86, e51184 (2014).

[9] J. D. White, R. E. Scholten, "Compact diffraction grating laser wavemeter with sub-picometer accuracy and picowatt sensitivity using a webcam imaging sensor," Rev. Sci. Instrum. 83(11), 113104, (2012).

[10] M. A. Bani, M. Nazeri, et al., "Studying Tunability of Some NIR Semiconductor Lasers by External Cavity Setup" Proc. of SPIE 10453, inthe III International Conference on Applications in Optics and Photonics (AOP), Faro, Portugal (2017).

[11] H. Abbasi, M. Nazeri, et al., "Studying Tunability of 780 and $655 \mathrm{~nm}$ Semiconductor Lasers in Littrow Configuration" The 21st Iranian Conference on Optics and Photonics (ICOP2015), Tehran, Iran (2015).

[12] B. L. Volodin, S. V. Dolgy, et al. "Wavelength stabilization and spectrum narrowing of high-power multimode laser diodes and arrays by use of volume Bragg gratings," Opt. Lett. 29(16), 1891-1893 (2004).

[13] Weitkamp, Claus, ed. Lidar: range-resolved optical remote sensing of the atmosphere. Vol. 102. Springer Science \& Business, (2006). 
[14] T. W. Hänsch, "Repetitively pulsed tunable dye laser for high resolution spectroscopy," App. Opt. 11, 895-898 (1972).

[15] M. G. Littman, H. J. Metcalf, "Spectrally narrow pulsed dye laser without beam expander," App. Opt. 17, 2224-2227 (1978).

[16] C. J. Hawthorn, K. P. Weber, "Scholten, R. E., Littrow configuration tunable external cavity diode laser with fixed direction output beam," Rev. Sci. Instrum. 72(12), 4477-4479 (2001).

[17] I. Park, C. Sydlo, et al. "Generation and spectroscopic application of tunable continuous-wave terahertz radiation using a dual-mode semiconductor laser," Meas. Sci. Technol. 19 (2008).

[18] A. Bagheri, M. Nazeri, et al. "Design and Construction of a Spectrometer with $0.04 \mathrm{~nm}$ Resolution at 780 nm Central Wavelength," Optic and Photonic Conference, Tehran, Iran (2017).

\section{Introduction}

In the middle of the 20th century, finding the fact that a p-n junction can be used for generating coherence light led to a great revolution in laser industry and as a consequence in the related fields [1]. The smaller dimension and very lower weight compared to other types of lasers, as well as low power operation, desired efficiency, low price and good tunability range were the main advantage that made them a remarkable field of research. Along with the development of the sciences of physics, chemistry and electronics, the technology of this type of lasers has also improved. Developments in related fields such as deposition, power supplies and optical design led this type of lasers to become efficient and single mode lasers. This type of lasers can be used for various applications such as laser spectroscopy experiments, the telecommunications industry, a source for pumping other lasers, measuring and manipulating the quantum state of atoms, laser cooling and generation of terahertz radiation [2-11]. Nowadays, using lasers with varying wavelengths is an interesting field of research; therefore, lasers will turn more applicable when their wavelengths can be tuned. There are a number of tuning mechanisms; one of them is using an external cavity as a feedback section. The principle of laser wavelength tuning by use of external feedback typically includes a wavelength-selective device positioned in the optical path of the laser beam that feeds a narrow part of the laser emission spectrum back into the laser cavity. There is another type of external-cavity laser that uses a resonator based on an optical fiber rather than on free-space optics. Tunable external cavity diode lasers (ECDLs) have been broadly developed. They have different applications in various fields of science. [12]. Tunable external cavity diode lasers (ECDLs) usually use a diffraction grating as the wavelength-selective element in the external resonator. In ECDLSs, the first order of diffraction of the grating provides optical feedback to the laser diode chip. In this case, a diode chip is required with a bandgap producing sufficient gain at the desired wavelength. The typical laser diode will have gain over several nanometers. ECDLs will often simultaneously support two or more closely-spaced longitudinal cavity modes with the total output power split between modes such that the effective power is substantially reduced. ECDLs have replaced complex and expensive traditional dye and Titanium Sapphire lasers as the workhorse laser of atomic physics labs [8,9]. Moreover, dual wavelength Littman ECDLs are used for continuous terahertz radiation generations and also differential absorption spectroscopy [13]. The aim of this paper is to study tunability of four near-infrared semiconductor lasers with center wavelengths from $780 \mathrm{~nm}$ to $1000 \mathrm{~nm}$ in four different configurations (wavelength dependent and wavelength independent beam direction setup).

\section{Materials and Methods}

Due to the wide gain of semiconductor lasers, this type of lasers has a good ability for wavelength tuning. Many different configurations have been developed each having advantages and disadvantages. The most common configurations are the Littrow [14] and Littman [15] configurations while they are simple and applicable at the same time. In this paper, both Littrow and Littman configurations are used for tuning the lasers. A schematic diagram of single mode Littrow configuration is shown in figure 1.

As it has been shown in figure 1., the zeroth-order of the diffraction from the grating is used as the output beam of the tuned laser and the first-order of the diffraction from the grating is used as the feedback beam which is reflected back into the laser. In this setup, according to the angle between the laser and the diffraction grating, a specific wavelength is selected. Therefore, the output beam direction is wavelength dependent, leading to alignment problems when tuning the laser in some optical setups. The optical setup of single mode Littrow configuration is shown in figure 2 . 


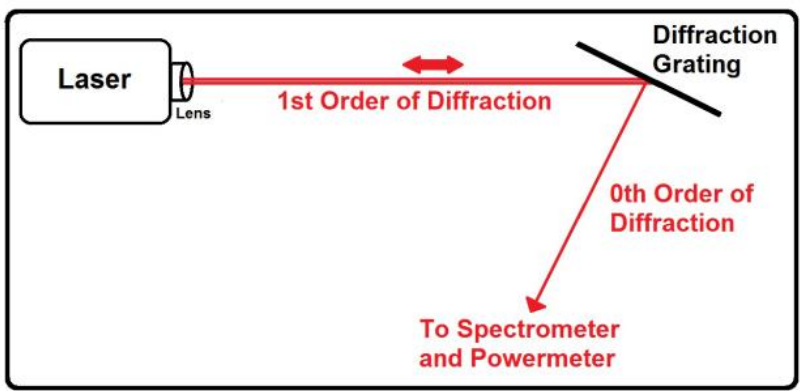

Fig. 1. A schematic diagram of single mode Littrow configuration.

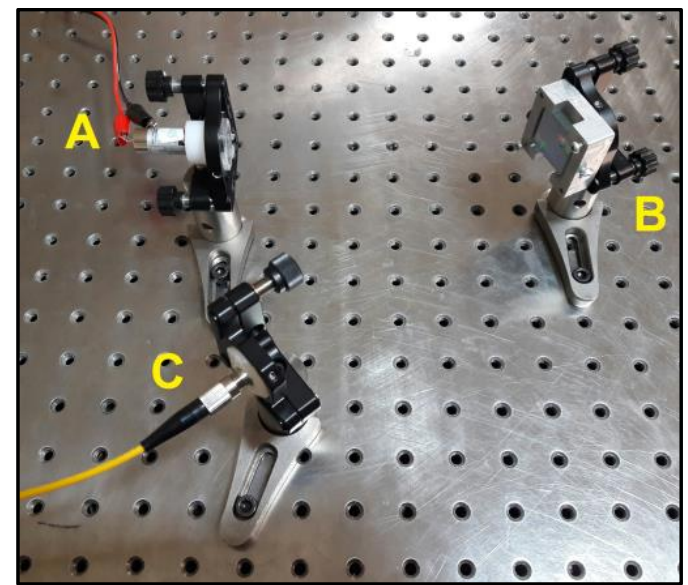

Fig. 2. Optical setup of single mode Littrow configuration, A: Laser diode, B: Diffraction grating and C: Collecting fiber optic.

Due to the specific application of $780 \mathrm{~nm}$ laser in continuous terahertz generation and also differential absorption spectroscopy [13], another type of external cavity tuning setup named dual mode (dual wavelength) has been developed. In this configuration, first, the initial output of the laser is separated into two beams by adding a beam splitter to the single mode Littorow setup, then each arm is tuned separately based on the rotation of the diffraction gratings. The wavelength of these two tuned modes can be either close or far from each other. The output power of dual mode setup is similar to the single mode one. The schematic setup of dual mode Littow configuration is shown in figure 3.

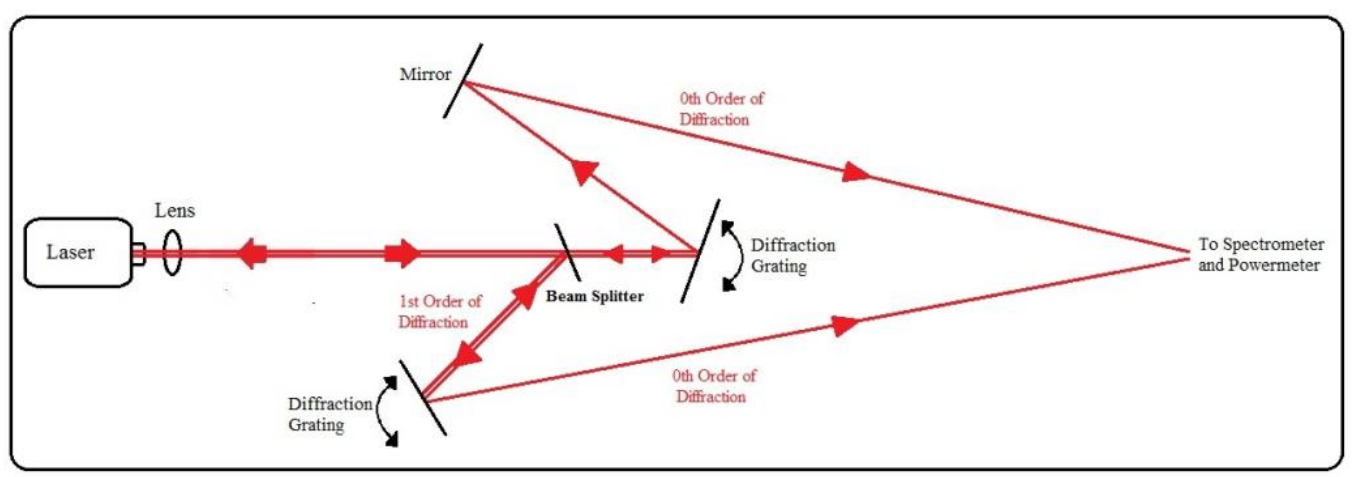

Fig. 3. A schematic diagram of dual mode Littrow configuration.

As shown in Fig. 3, the output beam of the laser is passing the 50-50 beam splitter after collimation using a lens. Each arm acts like a single mode setup. The first order of diffraction is used for tuning and zeroth order of that is used as an output. By adding an additional mirror to the way of one of the outputs the both outputs can be directed to the single optical fiber. The optical setup of dual Littrow setup is shown in Fig. 4. 


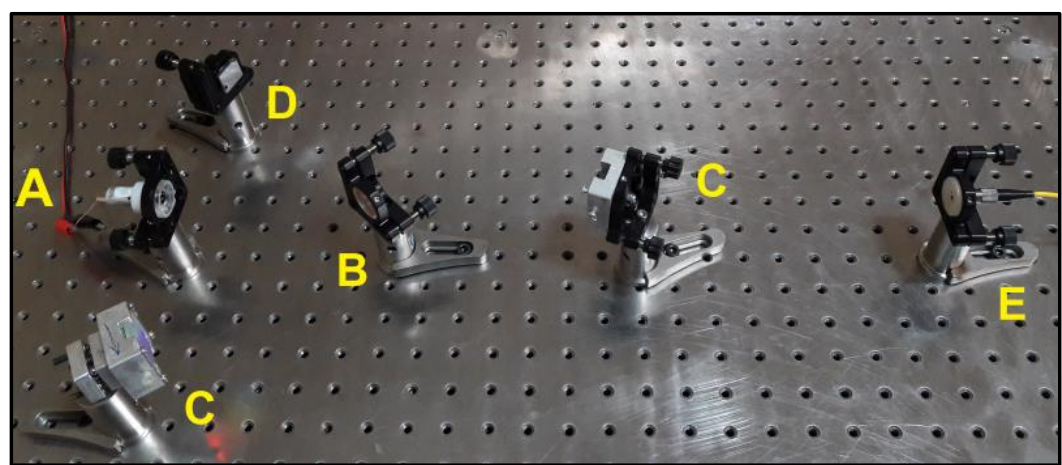

Fig. 4. Optical setup of dual mode Littrow configuration, A: Laser diode, B: Beam splitter, C: Diffraction gratings, D: Mirror, E: Collecting fiber optic.

If one needs wavelength independent beam direction, it can be achieved by adding an extra element, such as an intracavity beam splitter as an output coupler or a single plane mirror which is fixed relative to the tuning diffraction grating [16]. A schematic diagram of Littman configuration is shown in figure 5. The main difference between Littman and Littrow configurations is that the Littman configuration has an extra plane mirror compared to the Littrow configuration. In addition, the Littman configuration is more complex and requires a larger grating compared to the Littrow configuration [16].

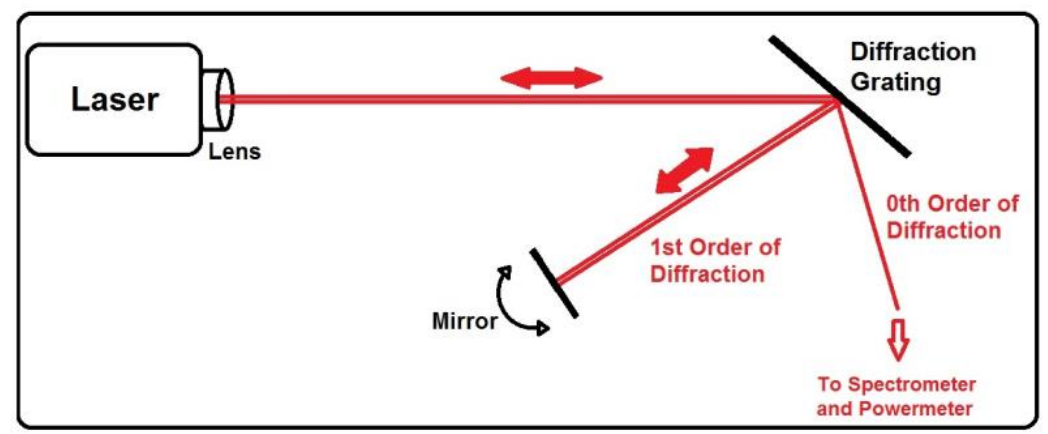

Fig. 5. A schematic diagram of single mode Littman configuration.

As it has been shown in figure 5, the Littman configuration uses a diffraction grating at near grazing incidence and an additional mirror. In this configuration, the first order of diffraction reflects back to the grating and the laser by the additional mirror. Therefore, in this setup, the wavelength is selected by the mirror angle, so that angle of the grating and the zeroth order of diffraction from the grating (the output beam) remain fixed during tuning the wavelength. The optical setup of single mode Littman configuration is shown in figure 6.

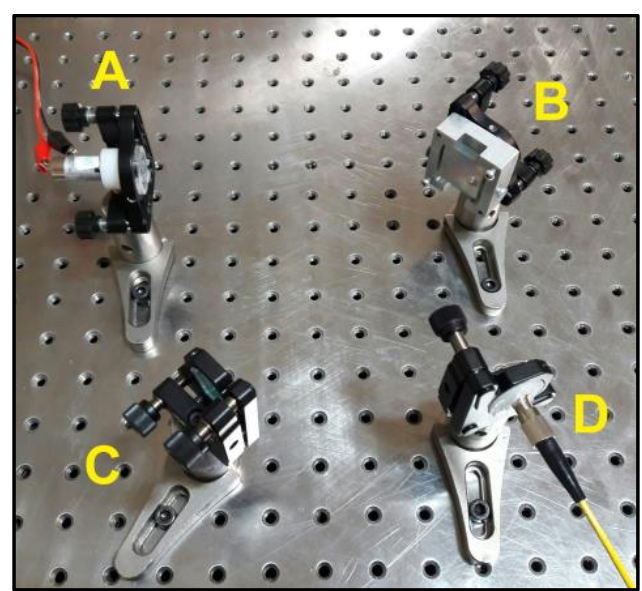

Fig. 6. Optical setup of single mode Littman configuration, A: Laser diode, B: Diffraction grating, C: Mirror, D: Collecting fiber optic.

Dual mode Littman configuration is developed by adding a beam splitter and a mirror to the single mode one. In this configuration, the wavelengths are selected by the angles of the mirrors, so that angle of the grating and the zeroth order of diffraction from the grating (the output beam) remain fixed during tuning 
the wavelength. Due to the fact that the first order of diffraction in this setup passes through two separated arms, this configuration is considered as two separate external cavity configuration. The wavelength of these two tuned modes can be either close or far from each other. While the output power of Littman setup is less than Littrow setup, this setup is used in the applications that need fixed position dual wavelength beams [17]. Figure 7 shows the schematic setup of the dual mode Littman configuration. The optical design of the dual mode Littman configuration is shown in figure 8.

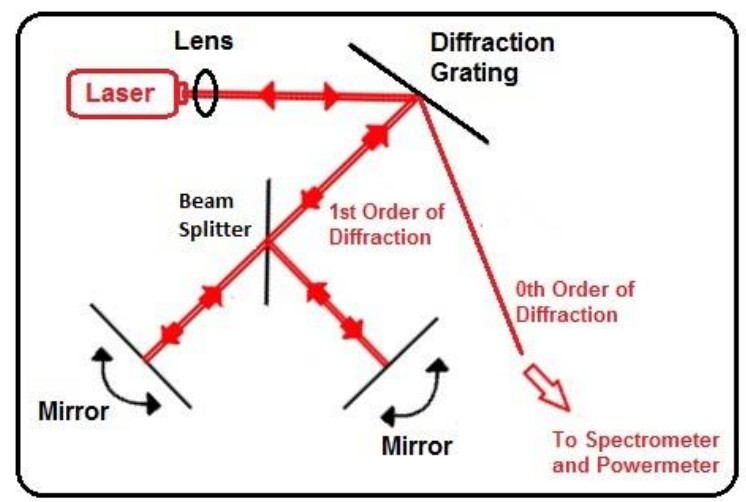

Fig. 7. A schematic diagram of dual mode Littman configuration.

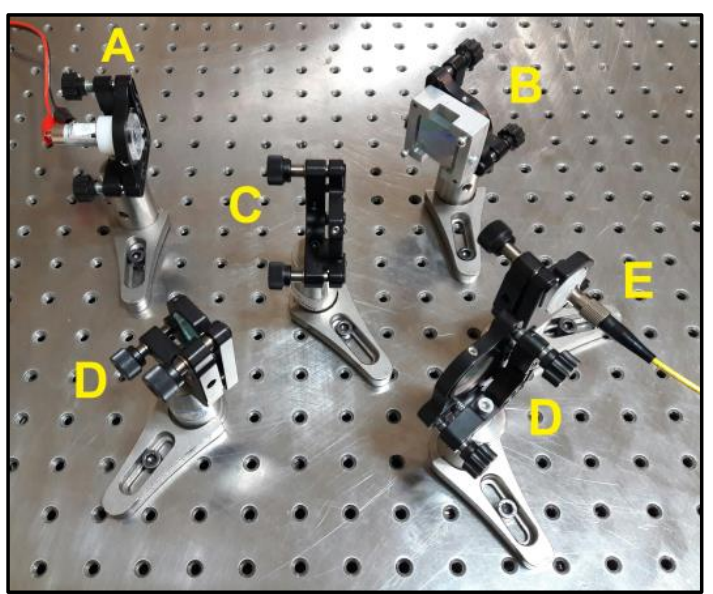

Fig. 8. Optical setup of dual mode Littman configuration, A: Laser diode, B: Diffraction grating, C: Beam splitter, D: Mirrors, E: Collecting fiber optic.

One can use a temperature sensor and a thermoelectric cooler to control the temperature. A plane front surface mirror (BB03-E03, Thorlabs) and a $1200 \mathrm{l} / \mathrm{mm}$ diffraction grating with blaze at $750 \mathrm{~nm}$ (GR251208, Thorlabs) have been used in this research. Kinematic mirror mount (KM100, Thorlabs), pedestal pillar post (RS3P/M, Thorlabs) together with clamping fork (CF125, Thorlabs) were used to hold the mirrors and gratings. The experiment has been carried out using four semiconductor lasers which typically lase at $783 \mathrm{~nm}(25 \mathrm{~mW}$, operating at $1.8 \mathrm{~V}$ and $30 \mathrm{~mA}$ current), $806.3 \mathrm{~nm}(8.4 \mathrm{~mW}$, operating at $2.5 \mathrm{~V}$ and $80 \mathrm{~mA}$ current), $888.9 \mathrm{~nm}(6.23 \mathrm{~mW}$, operating at $2.4 \mathrm{~V}$ and $20 \mathrm{~mA}$ current) and $980.6 \mathrm{~nm}(18.1 \mathrm{~mW}$, operating at $2.4 \mathrm{~V}$ and $50 \mathrm{~mA}$ current). The power of the laser was measured directly through the head of a digital optical power meter (Power Range: $50 \mathrm{nW}-50 \mathrm{~mW}$ ) and the wavelength of that was measured after passing the beam through the optical fiber (200 micron glass-core) of a compact CCD spectrometer (Spectral Range: 200 - $1000 \mathrm{~nm}$ ). In order to measure the spectrums in dual mode, an in-house spectrometer was designed and developed. In this spectrometer, a reflective diffraction grating is used as a dispersive element for spectral separation. Optical design of spectrometer was done by Zemax software. A 1200 groove/mm grating with blaze at $750 \mathrm{~nm}$ (GR50-1208, Thorlabs) and a concave mirror with $70 \mathrm{~cm}$ focal length (in house) were used in this spectrometer. The entrance of spectrometer was an optical fiber (P1780A-FC-1 for $783 \mathrm{~nm}$ laser and P1-830A-FC-1 for other lasers, both from Thorlabs) with FC/PC connection. A silicon linear array CCD with 3648 pixels (TCD1304, Toshiba) was used as a detector. By using a $9 \mu \mathrm{m}$ 
optical fiber the resolution of this spectrometer is $0.04 \mathrm{~nm}$ at $780 \mathrm{~nm}$ and its free spectral range is $27 \mathrm{~nm}$ [18]. Figures 9 and 10 show the internal and external picture of the developed spectrometer, respectively.

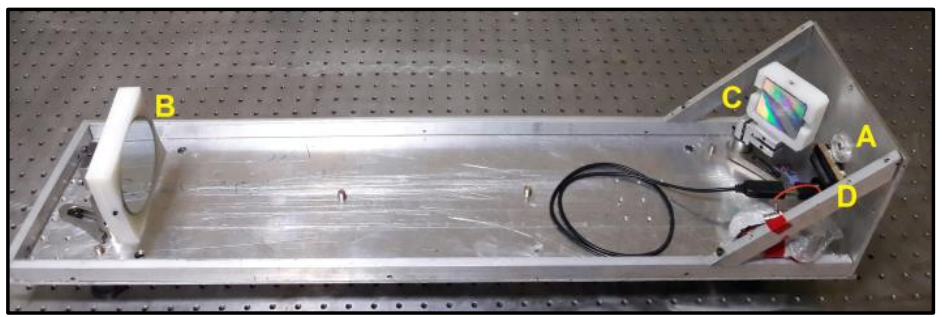

Fig. 9. Internal optical setup of the developed spectrometer, A: Fiber Input, B: Concave mirror, C: Diffraction grating, D: Detector (linear array CCD).

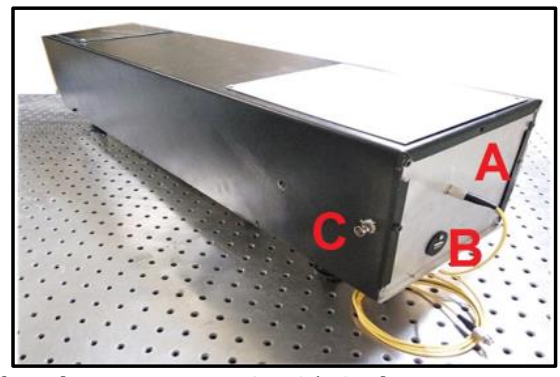

Fig. 10. External optical setup of the developed spectrometer, A: FC/PC Fiber optic connection, B: USB connection, C: Trigger Input.

\section{Result and Discussion}

The tuning range of $7.7 \mathrm{~nm}$ was demonstrated in Littrow configuration for the laser with center wavelength of $806.3 \mathrm{~nm}$. Figure 11 shows the output power of the laser diode with center wavelength of $806.3 \mathrm{~nm}$ in different wavelengths in Littrow configuration. Columns represent the power of the output beam of the laser in wavelengths of $801.5,802.3,8039,805.6,807.3,808.7$, and $809.2 \mathrm{~nm}$ respectively in $\mathrm{mW}$. The highest power achieved in this setup was $1.61 \mathrm{~mW}$ at $808.7 \mathrm{~nm}$.

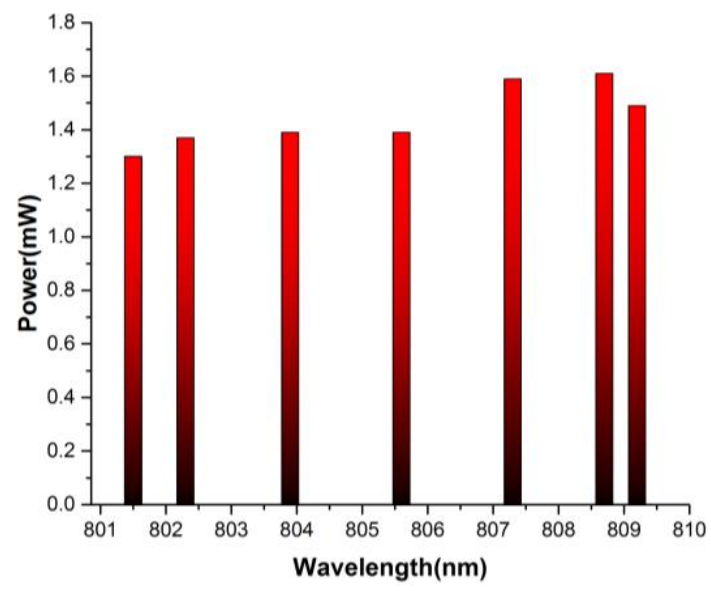

Fig. 11. The output power of the laser diode with center wavelength of $806.3 \mathrm{~nm}$ in different wavelengths in Littrow configuration.

In Littman configuration, the tuning range of $6.4 \mathrm{~nm}$ was demonstrated for the laser diode with center wavelength of $806.3 \mathrm{~nm}$. Figure 12 shows the output power of the laser diode with center wavelength of $806.3 \mathrm{~nm}$ in different wavelengths in Littman configuration. Columns represent the power of the output beam of the laser in wavelengths of 802.0, 804.6, 805.1, 807.0, 807.3, 808.2, and 808.4 nm respectively in $\mathrm{mW}$. The highest power achieved in this setup was $1.44 \mathrm{~mW}$ at $808.2 \mathrm{~nm}$. 


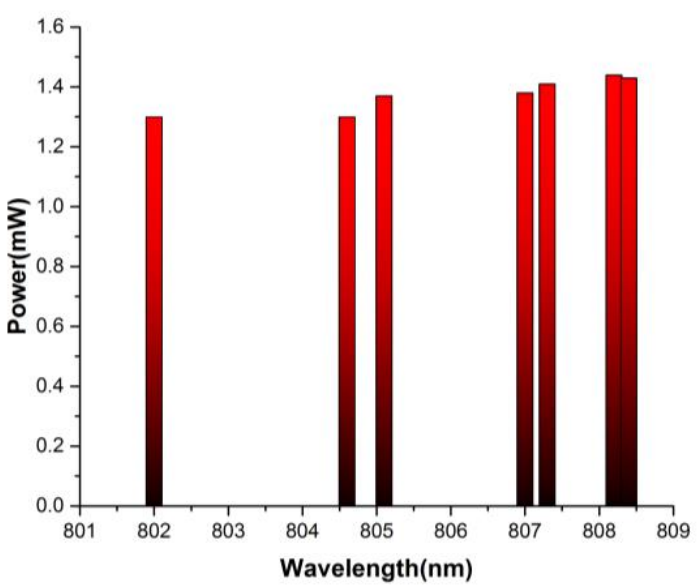

Fig. 12. The output power of the laser diode with center wavelength of $806.3 \mathrm{~nm}$ in different wavelengths in Littman configuration.

For the laser diode with center wavelength of $888.9 \mathrm{~nm}$, the tuning range of $15.4 \mathrm{~nm}$ was demonstrated in Littrow configuration. Figure 13 shows the output power of the laser diode with center wavelength of 888.9 $\mathrm{nm}$ in different wavelengths in Littrow configuration. In both Littrow and Littman setups, when the wavelength of the laser approaches to the end of the tuning range, suddenly the output beam jumps to the main wavelength of the laser. Columns represent the power of the output beam of the laser in wavelengths of $880.3,881.1,881.8,884.2,885.9,887.3,889.2,890.7,891.4,892.1,893.5,894.3$ and $895.7 \mathrm{~nm}$ respectively in $\mathrm{mW}$. As it is well visible in figure 13, the wavelengths near the main wavelength of the laser have more power. In this case, the highest power achieved in this setup was $0.91 \mathrm{~mW}$ at $889.2 \mathrm{~nm}$ which is very close to the main wavelength of the laser.

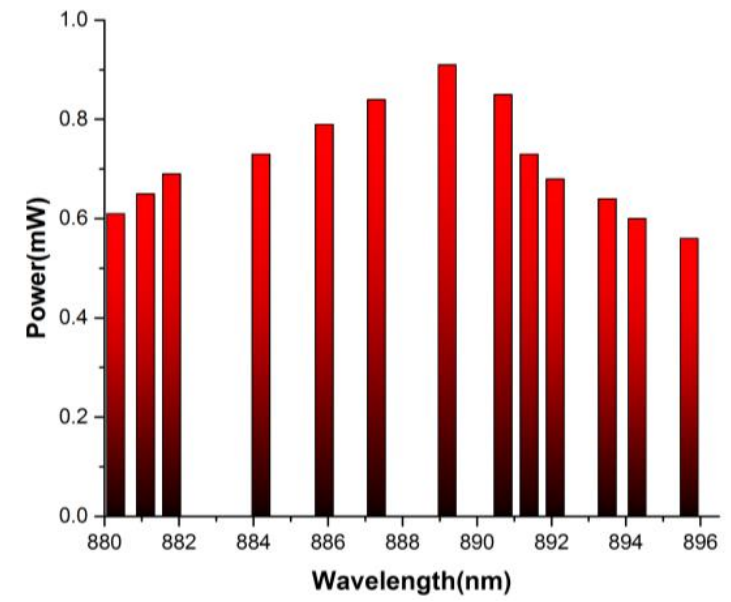

Fig. 13. The output power of the laser diode with center wavelength of $888.9 \mathrm{~nm}$ in different wavelengths in Littrow configuration.

In Littman configuration of the laser diode with center wavelength of $888.9 \mathrm{~nm}$, the tuning range of $11.2 \mathrm{~nm}$ was demonstrated. Figure 14 shows the output power of the laser diode with center wavelength of 888.9 $\mathrm{nm}$ in different wavelengths in Littman configuration. Columns represent the power of the output beam of the laser in wavelengths of 881.1, 881.3, 882.0, 882.7, 883.2, 884.2, 884.7, 885.4, 886.2, 887.1, 887.8, 889.3, $890.2,891.1$ and 892.3 respectively in $\mathrm{mW}$. The highest power achieved in this setup was $0.4 \mathrm{~mW}$ in 887.8 which is close to the main wavelength of the laser diode with center wavelength of $888.9 \mathrm{~nm}$. 


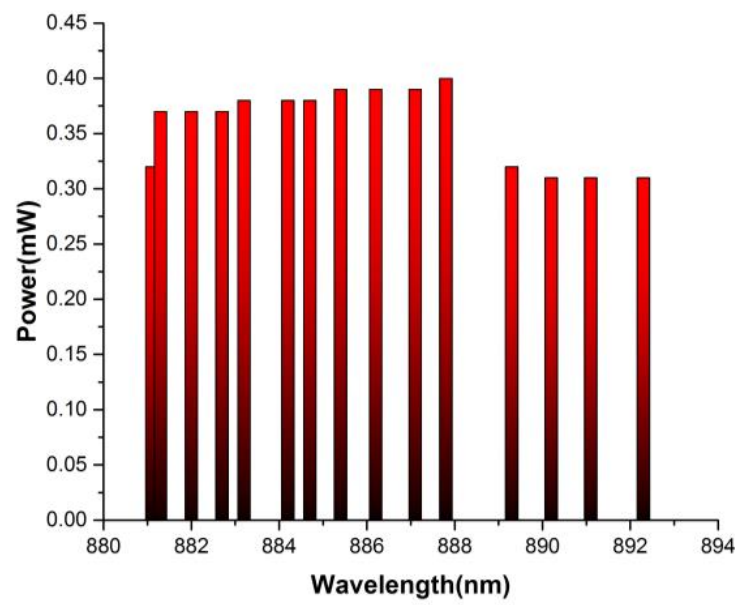

Fig. 14. The output power of the laser diode with center wavelength of $888.9 \mathrm{~nm}$ in different wavelengths in Littman configuration.

Finally, for the laser diode with center wavelength of $980.6 \mathrm{~nm}$, the tuning range of $13.0 \mathrm{~nm}$ was demonstrated in Littrow configuration. Figure 15 shows the output power of the laser diode with center wavelength of $980.6 \mathrm{~nm}$ in different wavelengths in Littrow configuration. Columns represent the power of the output beam of the laser in wavelengths of 971.7, 977.0,979.4, 980.2, 981.1,981.4, 984.0,984.3, and 984.7 respectively in $\mathrm{mW}$. As it can be seen in figure 15, the highest power achieved in this setup was 4.06 $\mathrm{mW}$ at $980.2 \mathrm{~nm}$ which is very close to the center wavelength of the laser diode with center wavelength of $980.6 \mathrm{~nm}$.

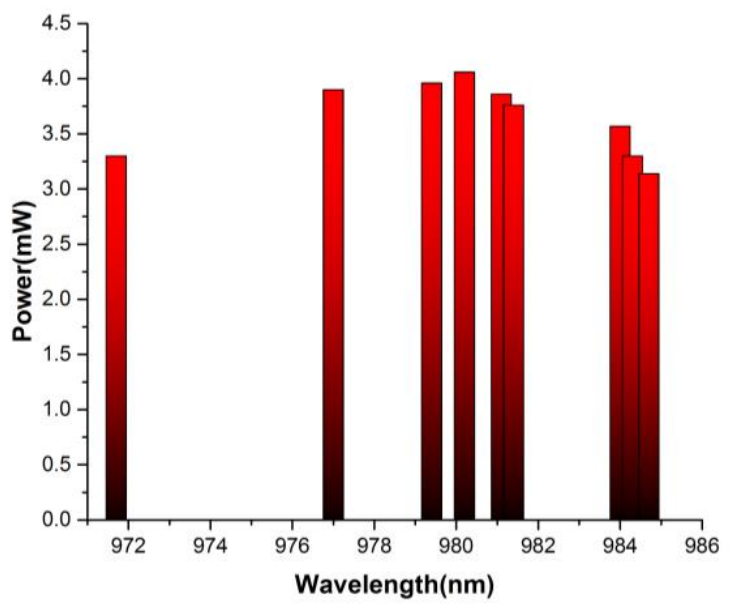

Fig. 15. The output power of the laser diode with center wavelength of $980.6 \mathrm{~nm}$ in different wavelengths in Littrow configuration.

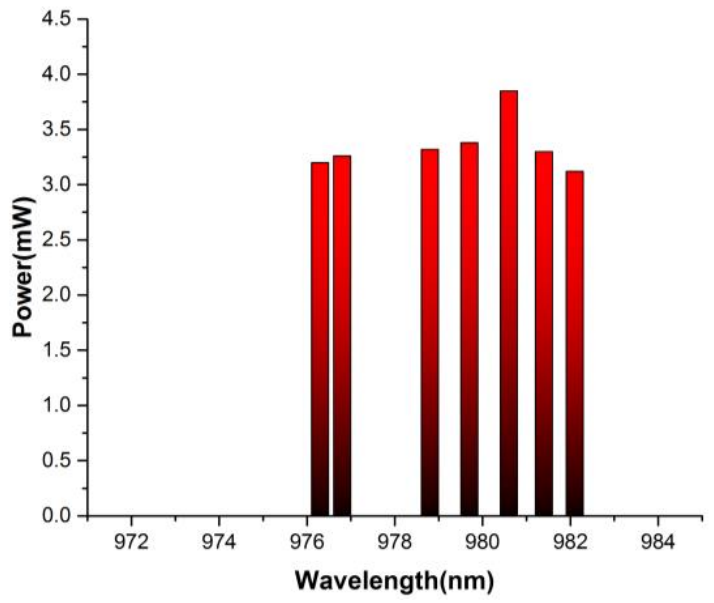

Fig. 16. The output power of the laser diode with center wavelength of $980.6 \mathrm{~nm}$ in different wavelengths in Littman configuration. 
In Littman configuration of the laser diode with center wavelength of $980.6 \mathrm{~nm}$, the tuning range of $5.5 \mathrm{~nm}$ was demonstrated. Figure 16 shows the output power of the laser diode with center wavelength of 980.6 $\mathrm{nm}$ in different wavelengths in Littman configuration. Columns represent the power of the output beam of the laser in wavelengths of $976.3,976.8,978.8,979.7,980.6,981.4$, and $982.1 \mathrm{~nm}$ respectively in $\mathrm{mW}$. As it can be seen in figure 16, the highest power achieved in this setup was $3.85 \mathrm{~mW}$ at $980.6 \mathrm{~nm}$ which is very close to the main wavelength of the laser diode with center wavelength of $980.6 \mathrm{~nm}$.

The tuning range of $7.1 \mathrm{~nm}$ was demonstrated in single mode Littrow configuration for the laser with center wavelength of $783 \mathrm{~nm}$. Figure 17 shows the output power of the laser diode with center wavelength of 783 $\mathrm{nm}$ in different wavelengths in single mode Littrow configuration. The intensity of each peak represents the power of the output beam of the laser in wavelengths of 779.3, 780, 780.4, 781.3, 781.5, 782.4, 782.7, 783.1, 783.7, 784.3, 784.7, 785.1, 785.6, 785.8, 785.9 and $786.4 \mathrm{~nm}$ respectively in $\mathrm{mW}$. The highest power achieved in this setup was $6.2 \mathrm{~mW}$ at $783.1 \mathrm{~nm}$.

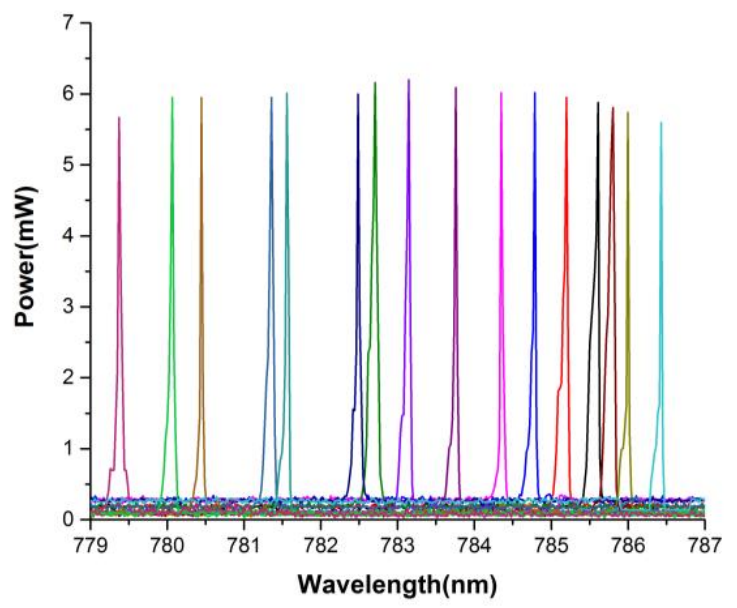

Fig. 17. The output power of the laser diode with center wavelength of $783 \mathrm{~nm}$ in different wavelengths in single mode Littrow configuration.

The tuning range of $7.4 \mathrm{~nm}$ was demonstrated in dual mode Littrow configuration of the laser with center wavelength of $783 \mathrm{~nm}$. Figure 18 shows the output power of the laser diode with center wavelength of 783 $\mathrm{nm}$ in different wavelengths in dual mode Littrow configuration. The intensity of each peak represents the power of the output beam of the laser in wavelengths of 777.9, 778.9, 780, 780.8, 781.73, 781.8, 782.8, 784, 784.9 and $785.3 \mathrm{~nm}$ respectively in $\mathrm{mW}$. The highest power achieved in this setup was $6.1 \mathrm{~mW}$ at $782.8 \mathrm{~nm}$. In figure 18, the closet achieved dual mode is related the red peaks and the farthest one is related to the pinkish peaks. Figures 19 and 20 show the closest and farthest achieved dual wavelength, respectively.

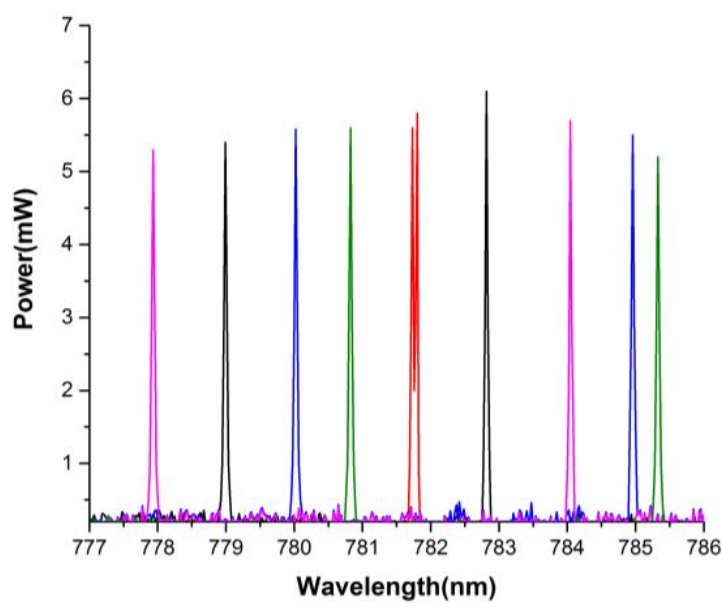

Fig. 18. The output power of the laser diode with center wavelength of $783 \mathrm{~nm}$ in different wavelengths in dual mode Littrow configuration. 


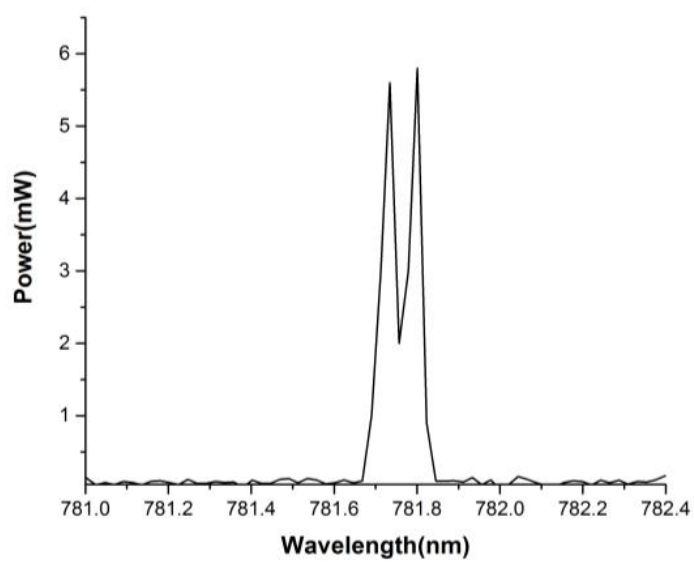

Fig. 19. The closet achieved dual mode in Littrow configuration for the laser diode with center wavelength of $783 \mathrm{~nm}$.

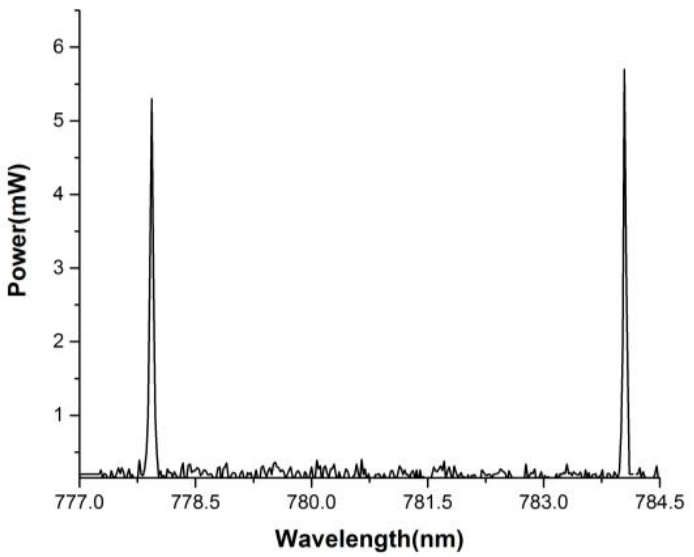

Fig. 20. The farthest achieved dual mode in Littrow configuration for the laser diode with center wavelength of $783 \mathrm{~nm}$.

As it is clear in the figures 19 and 20, the distance between the closest achieved wavelengths in dual mode Littrow configuration was $0.07 \mathrm{~nm}$ from $781.73 \mathrm{~nm}$ to $781.8 \mathrm{~nm}$, and the distance between the farthest one was $6.11 \mathrm{~nm}$ from $777.9 \mathrm{~nm}$ to $784 \mathrm{~nm}$.

The tuning range of $6.69 \mathrm{~nm}$ was demonstrated in single mode Littman configuration for the laser with center wavelength of $783 \mathrm{~nm}$. Figure 21 shows the output power of the laser diode with center wavelength of $783 \mathrm{~nm}$ in different wavelengths in Littman configuration. The intensity of each peak represents the power of the output beam of the laser in wavelengths of 778.8, 779.3, 779.7, 780.4, 781, 781.8, 782.5, 782.8, $783.1,783.4,784,784.6,785.3$ and $785.5 \mathrm{~nm}$ respectively in $\mathrm{mW}$. The highest power achieved in this setup was $5.8 \mathrm{~mW}$ at $783.1 \mathrm{~nm}$.

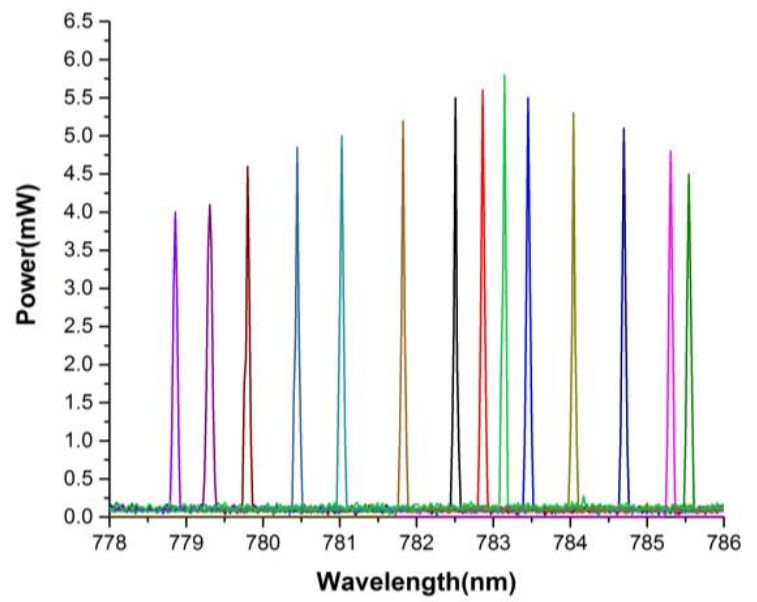

Fig. 21. The output power of the laser diode with center wavelength of $783 \mathrm{~nm}$ in different wavelengths in Littman configuration. 
The tuning range of $7.4 \mathrm{~nm}$ was demonstrated in dual mode Littman configuration for the laser with center wavelength of $783 \mathrm{~nm}$. Figure 22 shows the output power of the laser diode with center wavelength of 783 $\mathrm{nm}$ in different wavelengths in dual mode Littman configuration. The intensity of each peak represents the power of the output beam of the laser in wavelengths of 780.8, 781.03, 781.5, 781.8, 782.1, 782.3, 782.5, $782.8,783.05,783.11,783.4,783.6,783.8,784.05,784.2$ and $784.4 \mathrm{~nm}$ respectively in mW. The highest power achieved in this setup was $5.1 \mathrm{~mW}$ at $783.05 \mathrm{~nm}$. In figure 22, the closet achieved dual mode is related the red peaks and the farthest one is related to the dark blue peaks. Figures 19 and 20 show the closest and farthest achieved dual wavelength, respectively.

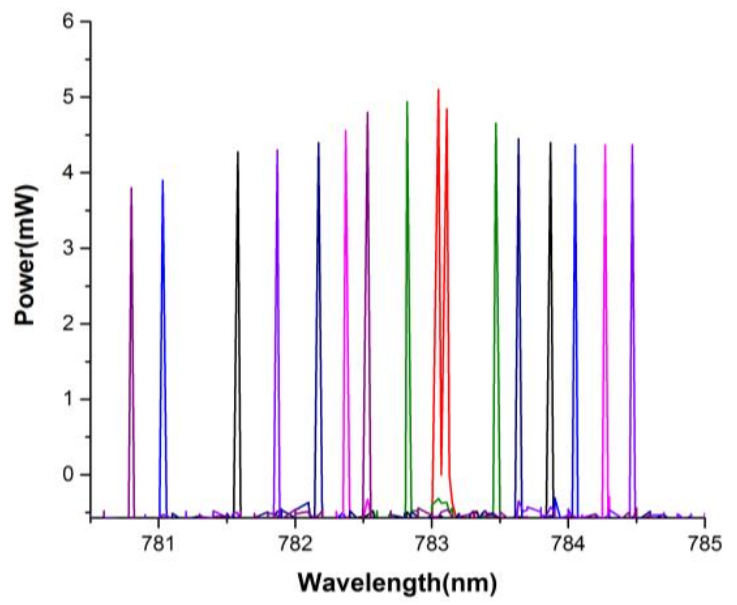

Fig. 22. The output power of the laser diode with center wavelength of $783 \mathrm{~nm}$ in different wavelengths in dual mode Littman configuration.

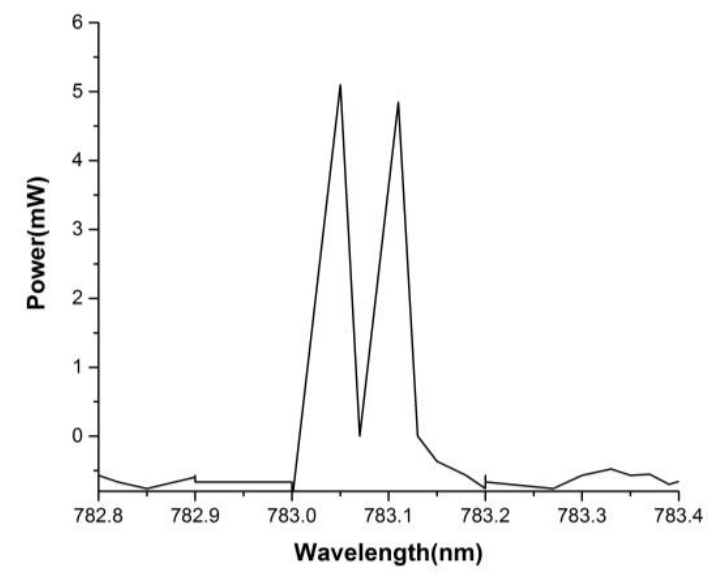

Fig. 23. The closet achieved dual mode in Littman configuration for the laser diode with center wavelength of $783 \mathrm{~nm}$.

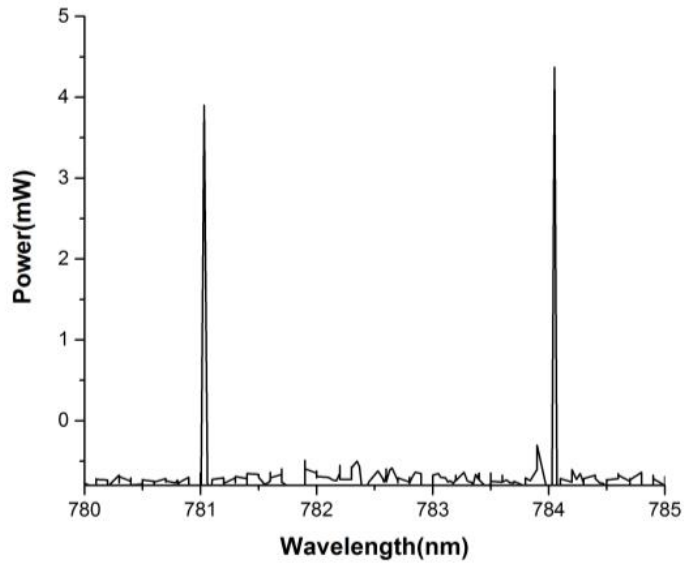

Fig. 24. The farthest achieved dual mode in Littman configuration for the laser diode with center wavelength of $783 \mathrm{~nm}$. 
As it is depicted in the figures 23 and 24, the distance between the closest achieved wavelengths in dual mode dual mode Littman configuration was $0.06 \mathrm{~nm}$ from $783.05 \mathrm{~nm}$ to $783.11 \mathrm{~nm}$, and the distance between the farthest one was $3.02 \mathrm{~nm}$ from $781.03 \mathrm{~nm}$ to 784.05 .

Additionally, the speed of tuning was tested in Littman configuration. A motorized actuator (Z812, Thorlabs) controlled by a DC servo motor controller (TDC001, Thorlabs) was connected to the rotating mirror of Littman setup in figure 5 to control the angle of mirror precisely and fast. Figure 25 shows the optical setup with the employed motorized actuator. As it is clear from the figure, the motorized actuator was connected to one of the adjuster screws of the kinematic mount to tilt the mirror precisely. Figure 26 shows the result of tuning with the motorized actuator. All achieved peaks were separated by the distance of $0.35 \mathrm{~nm}$. In this setup, the tuning range of $6.5 \mathrm{~nm}$ was achieved in $6 \mathrm{~s}$. So the tuning speed is below $1 \mathrm{~nm} / \mathrm{s}$ which is faster than the previous report by Park et al. [17].

Moreover, the effect of tuning on spectral bandwidth of the laser was investigated. A Michelson-Morley interferometer was used to measure the coherence length of the laser before and after tuning in single mode Littman setup. The coherence length of the laser was measured to be more than $3 \mathrm{~m}$ correspond to the bandwidth of $50 \mathrm{MHz}$; the same bandwidth was measured after tuning. Therefore, no effect on spectral bandwidth of laser was observed after tuning.

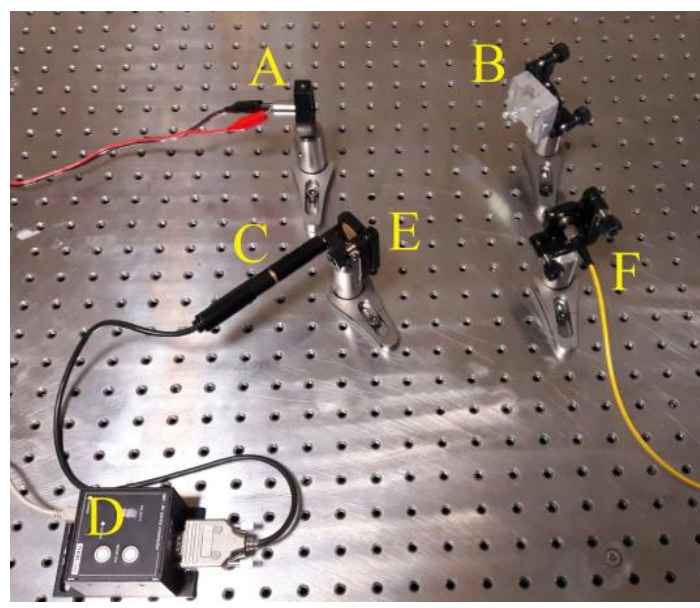

Fig. 25. The optical setup of single mode Littman configuration with the motorized actuator. A: Laser diode, B: Diffraction grating, C: Motorized actuator, D: DC servo motor controller, E: Mirror, F: Collecting fiber optic.

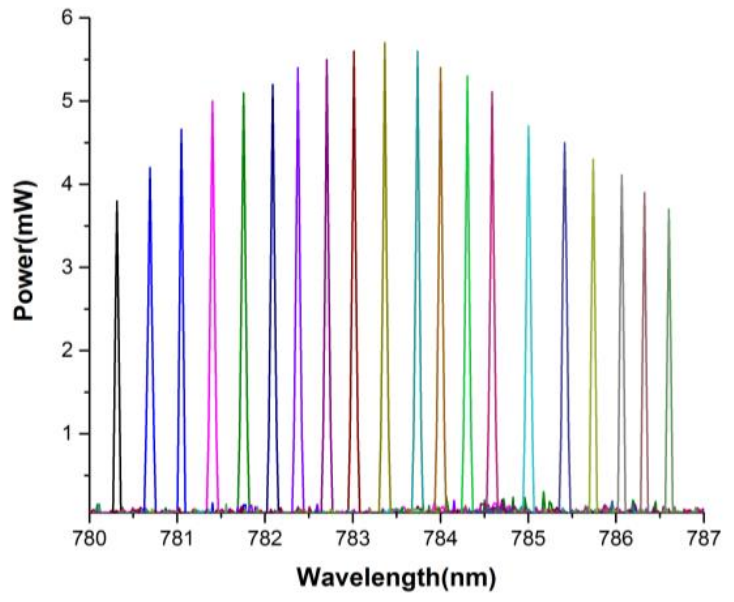

Fig. 26. The output power of the laser diode with center wavelength of $783 \mathrm{~nm}$ in different wavelengths in single mode Littman configuration connected to an actuator.

\section{Conclusions}

The tunability of four semiconductor lasers with center wavelengths from 780 to $1000 \mathrm{~nm}$ in two different setups has been studied. Both Littrow (wavelength dependent beam direction setup) and Littman 
(wavelength independent beam direction setup) configurations have been employed in this experiment. The wavelength of the output beam of the laser has been measured by means of a grating spectrometer and the power of the output beam has been measured by means of a power meter. In all four cases, both configurations showed a good tuning range, but Littrow configuration shows a little better tuning range. The tuning ranges of 7.1 and $6.69 \mathrm{~nm}$ for the laser diode with center wavelength of $783 \mathrm{~nm}$, the tuning ranges of 7.7 and $6.4 \mathrm{~nm}$ for the laser diode with center wavelength of $806.3 \mathrm{~nm}$, the tuning range of 15.4 and $11.2 \mathrm{~nm}$ for the laser diode with center wavelength of $888.9 \mathrm{~nm}$ and finally the tuning range of 13.0 and $5.5 \mathrm{~nm}$ for the laser diode with center wavelength of $980.6 \mathrm{~nm}$ were demonstrated in Littrow and Littman configurations, respectively. Moreover, the tunability of $783 \mathrm{~nm}$ laser in dual mode tuning is studied in both Littrow and Litman configurations. The tuning range of 7.4 and $3.67 \mathrm{~nm}$ were demonstrated in dual mode Littrow and dual mode Littman configuration for the laser diode with center wavelength of $783 \mathrm{~nm}$, respectively. The distance between the closest and farthest achieved wavelengths in dual mode Littrow configuration was $0.07 \mathrm{~nm}$, and $6.11 \mathrm{~nm}$, respectively. The distance between the closest and farthest achieved wavelengths in dual mode dual mode Littman configuration was $0.06 \mathrm{~nm}$ and $3.02 \mathrm{~nm}$, respectively. In both Littrow and Littman setups, when the wavelength of the laser approaches to the end of the tuning range, suddenly the output beam jumped to the main wavelength of the laser. In both Littrow and Littman setups of all experiments, the wavelengths near the main wavelength of the laser had more power. The highest achieved power of these lasers in the Littrow setup was more than that of the Littman setup which is probably related to the loss in the reflection of an extra mirror and some misalignment problems. The tuning speed was measured to be $1 \mathrm{~nm} / \mathrm{s}$. Also, no effect on spectral bandwidth of the laser was observed. 